\title{
Construction of 3D Dynamic Statistical Deformable Models for Complex Topological Shapes
}

\author{
Paramate Horkaew and Guang-Zhong Yang \\ Royal Society/Wolfson Foundation MIC Laboratory, \\ Department of Computing, \\ Imperial College of Science, Technology and Medicine, London, SW7 2BZ, UK \\ \{phorkaew, g.z.yang\}@imperial.ac.uk \\ http://vip.doc.imperial.ac.uk
}

\begin{abstract}
This paper describes the construction of 3D dynamic statistical deformable models for complex topological shapes. It significantly extents the existing framework in that surfaces with higher genus can be effectively modeled. Criteria based on surface conformality and minimum description length is used to simultaneously identify the intrinsic global correspondence of the training data. The proposed method requires neither surface partitioning nor artificial grids on the parameterization manifold. The strength of the method is demonstrated by building a statistical model of the complex anatomical structure of the left side of human heart that includes the left ventricle, left atrium, aortic outflow tract, and pulmonary veins. The analysis of variance and leave-one-out-cross-validation indicate that the derived model not only captures physiologically plausible modes of variation but also is robust and concise, thus greatly enhancing its potential clinical value.
\end{abstract}

\section{Introduction}

With the increasing popularity of the Active Shape and Appearance Models [1] [2], 3D shape modeling and segmentation based on these techniques are gaining significant clinical interest. In essence, the technique recovers the underlying shape by exploiting a priori knowledge about the plausible variations of anatomical structures captured by the Point Distribution Model (PDM). The practical quality of a PDM relies on the definition of correspondence across a set of segmented samples. Brett and Taylor 3] generated correspondences using ICP while a diffeomorphism between the shapes was maintained by harmonic map. Davies et al. 4 solved this problem by manipulating global parameterization subject to the Minimum Description Length (MDL) criterion. For time varying $3 \mathrm{D}$ objects, identification of dense correspondence within the training set is a significant challenge. Landmark techniques based on anatomical features has been proven to be problematic for 3D shapes that undergo large deformation over time. To resolve this difficulty, Horkaew and Yang recently proposed a harmonic embedding technique for establishing optimal global correspondence for a set of 
dynamic surfaces whose topological realization is homeomorphic to a compact 2D manifold with boundary [5]. Its application to left ventricular modeling has demonstrated its compactness and ability for capturing principal modes of variation that are biologically meaningful.

One of the key challenges of 3D statistical shape modeling is the parameterization of surfaces with generic topologies. For high genus shapes, most of the current techniques 678 have limited their investigation to surface remeshing, texture mapping and metamorphosis between a pair of objects. The method recently proposed by Praun et al. 9, suggests a parameterization algorithm based on heuristic legal checks to avoid path intersection for a group of surfaces. Although being able to create a valid set of smooth surface partitions, the resultant meshes are not necessarily statistically optimal. This is also true for other approaches [10 11]. The alternatives of using level set embedded in higher dimension manifold or volumetric registration, on the other hand, are less compact and do not explicitly guarantee the topology of the final result [12]13].

General anatomical structures can have shapes with higher genuses. A normal human heart as shown in Figure 1, for example, consists of four chambers, each topologically equivalent to a sphere with different numbers of boundaries corresponding to valvular and vascular orifices. The left and right ventricles are connected with the atria at mitral and tricuspid valves. When all the valvular structures and inflow/outflow tracts are considered, the shape to be modeled becomes highly complex even in its static form. The purpose of this paper is to extend the harmonic embedding techniques proposed by Horkaew and Yang for anatomical structures with complex topology. One possible extension to the technique is to use surface subdivision such that each surface patch can be harmonically embedded on its own. This, however, requires the identification of geometrical landmarks for separating the overall structure into physiologically independent but topologically simple substructures. The network of partitioning curves implies the initial boundary correspondence and surface subdivision. For dynamic shapes, the treatment of surface continuity and correspondence across boundaries with this approach, however, is challenging. In this paper, a more effective embedding scheme that incorporates multiple boundaries associated with high genus objects is proposed. Whilst ensuring the generative PDM is geometrically and statistically optimal, the method also has the following features:

- No surface partitioning prior to parameterization is required while maintaining the conformal structure and multi-resolution properties for the surface.

- No artificial structure is introduced to the parameterization domain, i.e., the correspondence is implicit to ensure flexibility in mathematical treatment and reduce shape artifacts.

We demonstrate in this paper how the proposed method can be used for modeling the entire left side of the heart including Left Atrium (LA), Left Ventricle (LV), major Pulmonary Veins (PV) and the Left Ventricular Outflow Tract (LVOT). 


\section{Material and Methods}

\subsection{Surface Parameterization}

One of the key steps for the proposed statistical shape modeling scheme is the parameterization of a generic surface with its equivalent topological domain. The base domain used in this study is a unit sphere. Since the underlying anatomy of LV and LA across subjects is consistent, the vertices correspond to the boundary orifices are mapped onto given locations of the base domain before surface parameterization. For LV and LA, the mitral annulus was parameterized onto a contour connecting the unit spheres used for mapping the LA and LV, as shown in Figure 1(c). Without loss of generality, the mitral annulus was always parameterized onto a contour centered by the north pole so as to ensure that no singularity was introduced by mapping infinitesimal area of the surface to infinity. During the subsequent analysis the $G^{1}$ continuity across the mitral valve boundary was maintained by conformal parameterization.
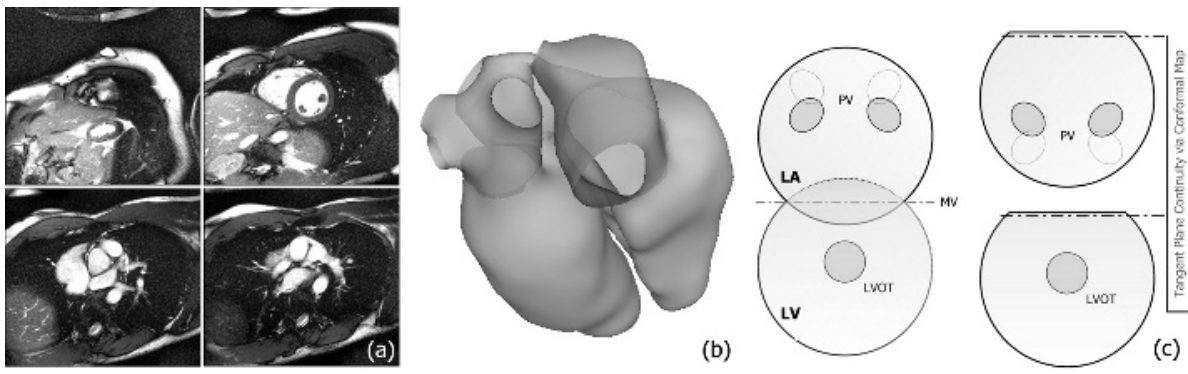

Fig. 1. Short axis MR images covering 4 chambers of the heart from the apex to the atria during diastole (a) and its corresponding 3D surface reconstruction (b). (c) The configuration used for surface parameterization of the LV and LA used for this study

The remaining internal vertices were then uniformly distributed on each sphere by using Tutte's mapping [14. With the introduction of boundary contours as shown in Figure 1(c), the trimmed sphere is no longer convex and mesh folding is inevitable. To alleviate this problem, the vertices were first mapped to a stereographic complex domain followed by iterative mesh untangling and smoothing [15. The technique was based on maximizing the minimum area of simplicial elements in a local sub-mesh such that

$$
\hat{\mathbf{x}}=\max _{\mathbf{x}}\left(\min _{1 \leq i \leq N}\left|\mathbf{x}_{i}^{1}-\mathbf{x} \mathbf{x}_{i}^{2}-\mathbf{x}\right|\right)
$$

In the above equation, the $i^{\text {th }}$ triangle in the local submesh is defined by the positions of the free vertex $\mathbf{x}$ and the positions of the two other vertices $\mathbf{x}_{i}^{1}$ and $\mathbf{x}_{i}^{2}$, and $\hat{\mathbf{x}}$ is the new optimal position of the free vertex. In this study, PolakRibiere's conjugate gradient optimization was adopted. This unfolding scheme 
results in topologically valid mesh that can be spatially degenerated. A variant of constrained Laplacian smoothing was subsequently applied to enhance mesh quality. The algorithm relocates the free vertex to the geometric center of the immediate neighboring vertices, only if the quality of the local submesh was improved according to the quality metric given by [16],

$$
\begin{aligned}
\alpha\left(T_{x}\right) & =(I) 2 \sqrt{3} \frac{\left\|\left(\mathbf{x}_{2}-\mathbf{x}_{0}\right) \times\left(\mathbf{x}_{1}-\mathbf{x}_{0}\right)\right\|}{\left\|\mathbf{x}_{2}-\mathbf{x}_{0}\right\|^{2}+\left\|\mathbf{x}_{0}-\mathbf{x}_{1}\right\|^{2}+\left\|\mathbf{x}_{1}-\mathbf{x}_{2}\right\|^{2}} \\
I & =\left\{\begin{array}{l}
+1, \text { if }\left(\mathbf{x}_{2}-\mathbf{x}_{0}\right) \times\left(\mathbf{x}_{1}-\mathbf{x}_{0}\right) \cdot N_{x}>0 \\
-1, \text { otherwise }
\end{array}\right.
\end{aligned}
$$

Where $T_{x}$ is a triangle defined by three vertices $\mathbf{x}_{0}, \mathbf{x}_{1}$ and $\mathbf{x}_{2}$ and the surface normal vector $N_{x}$ is evaluated at the center of the triangle. The function $I$ captures the inversion of the element to prevent mesh folding.

\subsection{Variational Approach to Conformal Surface Embedding}

The mesh obtained above generally has a good quality but its parameterization may not preserve the local geometric property. The purpose of conformal embedding at this stage is to reparameterize the LV and LA surfaces $M$ to their respective topologically equivalent unit spheres $S$ with corresponding boundaries. A variational approach to piecewise linear Harmonic Maps was used [17. The advantage of the variational approach over the leastsquare ones 510$]$ is that the convex constraints on the manifolds may be relaxed, provided that the initial parameterization is already of valid topology. Let $(M, m) \in \mathbf{R}^{3}$ and $(S, s) \in \mathbf{R}^{3}$ be two diffeomorphic Riemannian manifolds and $\boldsymbol{\Phi}:(M, m) \rightarrow(S, s)$ is a conformal map, which minimizes metric dispersion. This metric can be represented as a sum of potential energy in a configuration of springs, each of which is placed along an edge of the triangulation. For this approach, we define linear Laplacian operator $\triangle_{P L}: C^{P L} \rightarrow C^{P L}$ on function $\boldsymbol{\Phi}$ to be

$$
\triangle_{P L} \boldsymbol{\Phi}=\sum_{\{i, j\} \in M} \kappa_{i j}(\boldsymbol{\Phi}(i)-\boldsymbol{\Phi}(j))
$$

where $\boldsymbol{\Phi}=\left(\phi_{x}, \phi_{y}, \phi_{z}\right)$ is a harmonic map from the surface mesh $M$ to a unit sphere $S$ and $\kappa$ is a spring constant, defined as a summation of cotangent weighting coefficients of its subtended angles of a given edge $(i, j)$. Since map $\boldsymbol{\Phi}$ is harmonics if it only has a normal component $(\triangle \boldsymbol{\Phi})^{\perp}$ and its tangent component $\triangle \boldsymbol{\Phi}$ is zero, therefore at critical point we have $\triangle \boldsymbol{\Phi} \rightarrow(\triangle \boldsymbol{\Phi})^{\perp}$. In this case, $\boldsymbol{\Phi}$ can be solved by iteratively displacing internal free vertices in the steepest gradient direction, i.e.,

$$
\begin{gathered}
\delta \boldsymbol{\Phi}=-D \boldsymbol{\Phi} \times \delta t \\
D \boldsymbol{\Phi}(i)=\triangle \boldsymbol{\Phi}(i)-(\triangle \boldsymbol{\Phi}(i))^{\perp} \text { and }(\triangle \boldsymbol{\Phi}(i))^{\perp}=\langle\triangle \boldsymbol{\Phi}(i), \mathbf{n}(\boldsymbol{\Phi}(i))\rangle \mathbf{n}(\boldsymbol{\Phi}(i))
\end{gathered}
$$

where $\langle$,$\rangle is inner product in \mathbf{R}^{3}$ and $\mathbf{n}(\mathbf{p})$ is a normal vector on $S$ at point $\mathbf{p}$. For a sufficiently small step size $\delta t$, the resultant parameterization is guaranteed to 
converge to the conformal approximation. Finally, Deluanay triangulation was used to remesh the conformal domain with equilateral triangles to ensure that the final mesh is topologically correct, angle-preserving, and not degenerated. It is worth noting here that the conformal property used here also ensures the shape consistency, i.e., the $G^{1}$ continuity from LV to LA across the mitral annulus.

\subsection{Identifying Optimal Correspondence}

The next step of building the statistical shape model is to establish optimum point correspondence within the training set. With the proposed framework, we formulate the correspondence problem as that of defining a set of dense vector fields, by which each surface in the training set is deformed. These vector fields are interpolated with radial basis splines whose control points are constrained around the defined boundaries. It is worth noting that by doing so, no artificial grids were imposed on the parameterization process. To reduce the computational cost, a $k$ - $d$ tree was used for sampling a deformed surface point on the parametric domain. Similar to previous studies, MDL was employed as the optimization criterion for the derived statistical model. It is well known that the MDL function is highly non-linear and can have multiple local minima, the optimization is therefore carried out in a hierarchical manner. At the coarsest parameterization level, the correspondence vector fields were deformed such that they minimized the Euclidean distances between a pair of surfaces. At the subsequent levels $L$, a set of $4^{L-1}$ spline control points and corresponding vector values were recursively inserted into the domain, with their positions determined by maximizing the multi-site geodesic distances on an individual surface. The parameterization was optimized subject to the MDL of the entire training set on a per-surface basis. The ordering, by which each sample was optimized, was randomly permutated before each iteration [18. Conjugate gradient method was used to identify the optimal deformation fields and finally, Principal Component Analysis (PCA) was applied to capture the mean shape and principal modes of variation.

\subsection{Data Acquisition and Validation}

Four healthy subjects, (mean age $=27$, range 25 -29 years) were studied in supine position with a Siemens Sonanta $1.5 \mathrm{~T}(40 \mathrm{mT} / \mathrm{m}, 200 \mathrm{mT} / \mathrm{m} / \mathrm{ms})$. A breath-hold retrospectively ECG-gated trueFISP cine sequence $(\mathrm{TE}=1.63 \mathrm{~ms}, \mathrm{TR}=3.26 \mathrm{~ms})$ was used. The effective temporal resolution was $22-30 \mathrm{~ms}$ and multiple parallel short axis images were acquired in two multislice groups, one to cover from the valvular plane to the apex of the LV with $10 \mathrm{~mm}$ increments, and the other from the aortic arch to the valvular plane in $5 \mathrm{~mm}$ increments, due to the geometrical complexity of the LA. The in-plane and through-plane image resolution used was $1.2 \mathrm{~mm} \times 1.2 \mathrm{~mm}$ and $7 \mathrm{~mm}$, respectively. The image segmentation scheme follows the method used in [5] for depicting the cardiac borders. For the LV, the mitral and aortic valve planes were manually defined along with the left ventricular in-flow and out-flow tracts. Similarly, the planes cutting through the pulmonary 
veins were also specified, giving a total of five boundary contours including the mitral annulus. A total of thirty-four shapes of the LV and LA were used for building the dynamic statistical shape model.

\section{Results}

Figs. 2(a) shows an example of the initial Tutte's mapping of the LA projected onto a stereographic plane. It is evident that significant mesh folding is introduced particularly around the pulmonary veins. The resultant parameterization of the unfolded mesh after applying the proposed mesh smoothing and conformal parameterization are illustrated in Figs. 2 (b) and (c), respectively. A total of 10 control points, excluding those on the boundaries, as shown in Figs. 2(d) were used to interpolate correspondence vector fields to establish the optimal set of control points. It is worth noting that these vector fields were bounded on the manifold and determined by the MDL objective.
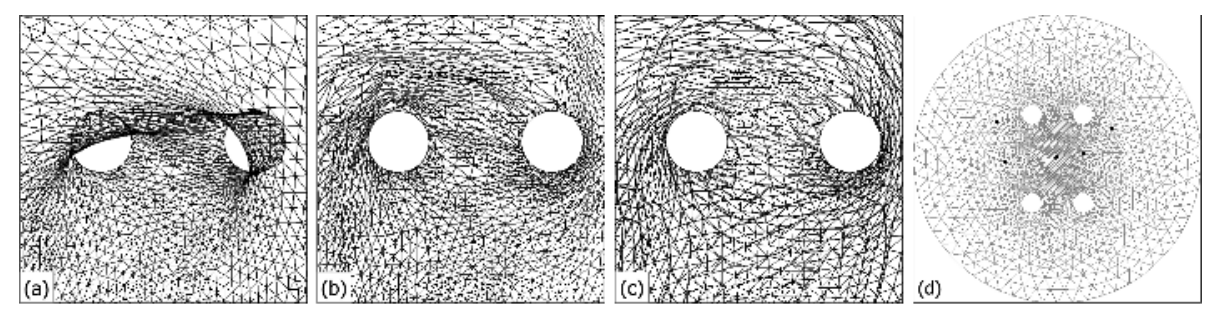

Fig. 2. The stereographic projection of the zoom up around the boundaries of the parameterized LA surfaces showing the initial state (a), after applying mesh untangling and smoothing (b) and conformal maps (c) and positions of the control points (d)

Fig. 3 demonstrates the principal modes of variation captured by the derived optimal PDM. An animation of the first three modes corresponds to contraction, radial twisting and shortening (http://vip.doc.ic.ac.uk/ gzy/osdm/). The compactness of a model is measured by using the total variance captured by the principal modes. The generalization ability is assessed by using leave-one-out reconstruction, i.e., the PDM was built by using both LV and LA surfaces from all but one subject and then fitted to the excluded samples. The accuracy of the reconstruction is measured as the residue of the fitted model to the actual data. The process is repeated by excluding each of the remaining subjects in turn. The averaged residue over the samples is then computed.

Fig. 4 shows the quantitative comparison of these measurements for the model obtained by sampling on the conformal map, and that obtained by minimizing Euclidean distance between a pair of surfaces, i.e., parameterization given at the first level of recursion, and the optimal model proposed in this paper. It can be observed from the graphs that the optimal model is $67 \%$ and $25 \%$, respectively, more compact than the conformal and minimized Euclidean models. 

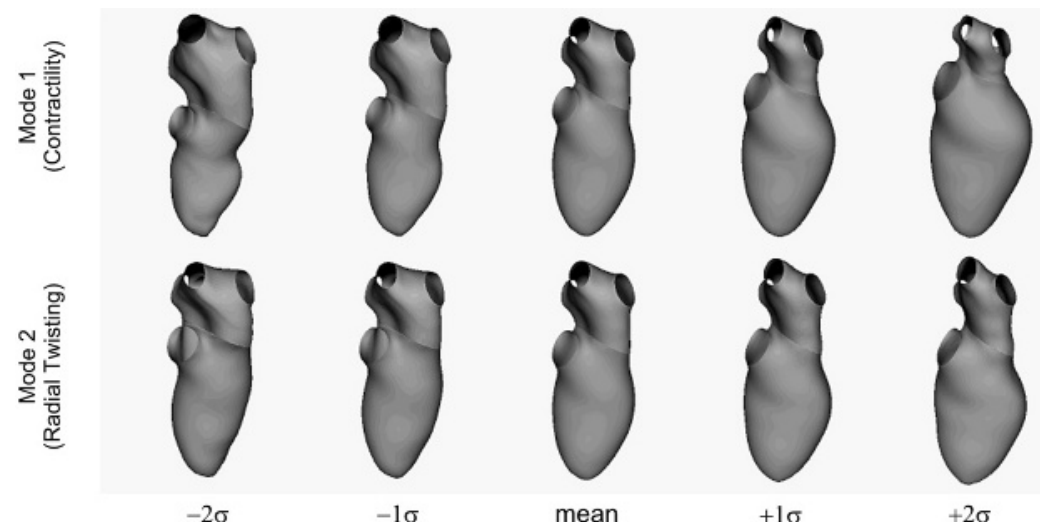

Fig. 3. The variation captured by the first two modes of variations. The shape parameters were varied by $\pm 2 \sigma$, seen within the training set. They corresponds to contraction and radial twisting
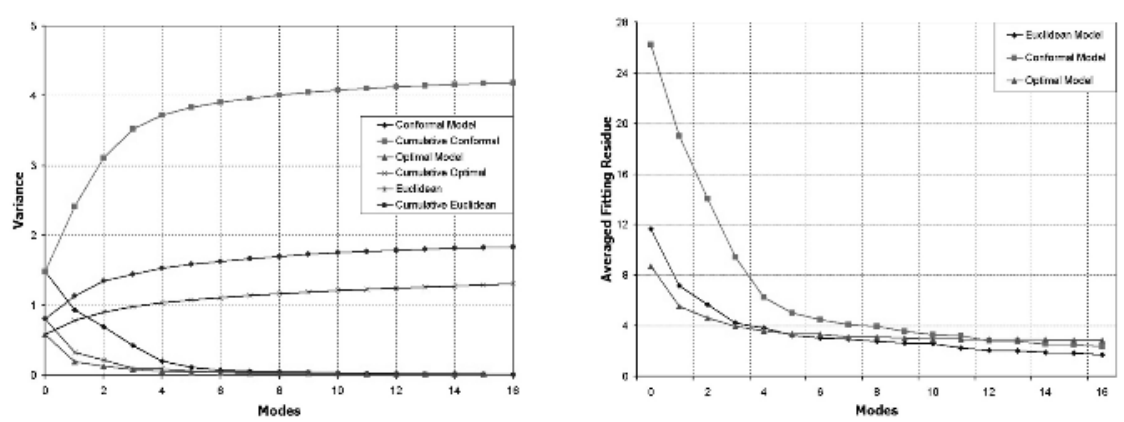

Fig. 4. Quantitative comparison of these measurements among the conformal, minimizing Euclidean distance, and the proposed optimal models, showing analysis of variances (left) and leave-one-out fitting residue (right)

Further analysis of the model shows that the averaged fitting residue of generated PDMs to the actual data by taking into account other modes of variation, the optimal model gives consistently less residue than that of the other methods.

\section{Discussions and Conclusion}

Conformal surface embedding is an essential step towards optimal statistical deformable surface modeling for dynamic structures. We have shown that the basic concept described in [5] can be extended to shapes with complex topology, thus enhancing its practical clinical value. The proposed method produces a PDM that is significantly more compact than the one obtained from the conformal and minimized Euclidean techniques. It must be pointed out, however, that the method proposed above applies only to a sub-class of all mathemati- 
cally plausible shapes and is not suitable for shapes such as n-torus. From our experience, however, the restriction imposed on our framework is realistic for modeling cardiovascular and other visceral structures.

\section{References}

1. T.F. Cootes, C.J. Taylor, D.H. Cooper and J. Graham: Active Shape Models Their Training and Application, CVIU, 61(1) (1995) 38-59.

2. T.F. Cootes, G.J. Edwards, and C. J. Taylor: Active Appearance Models, ECCV, 2 (1998) 484-498.

3. A.D. Brett and C.J. Taylor: Construction of 3D Shape Models of Femoral Articular Cartilage Using Harmonic Maps, MICCAI (2000) 1205-1214.

4. R.H. Davies, C.J. Twining, T.F Coots J.C. Waterton and C.J. Taylor: A Minimum Description Length Approach to Statistical Shape Modelling, IPMI (2001) 50-63.

5. P. Horkaew and G. Z. Yang: Optimal Deformable Surface Models for 3D Medical Image Analysis, IPMI (2003) 13-24.

6. M. S. Floater: Parametrization and Smooth Approximation of Surface Triangulations, CAGD, 14 (1997) 231-250.

7. S. Haker et al.: Conformal Surface Parameterization for Texture Mapping, IEEE Trans. Visualization and Computer Graphics, 6(2) (2000) 181-189.

8. B. Lévy and J. Mallet: Non-distorted Texture Mapping for Sheared Triangulated Meshes, SIGGRAPH (1998) 343-352.

9. E. Praun, W. Sweldens and P. Schroder: Consistent Mesh Parameterizations, SIGGRAPH (2001) 179-184.

10. M. Eck et al.: Multiresolution Analysis of Arbitrary Meshes, SIGGRAPH, (1995) 173-182.

11. O. Sorkine, D. Cohen-Or, R. Goldenthal and D. Lischinski: Bounded-distortion Piecewise Mesh Parameterization, IEEE Visualization (2002) 355-362.

12. A. Tsai et al.: Coupled Multi-Shape Model and Mutual Information for Medical Image Segmentation, IPMI (2003) 185-197.

13. A.F. Frangi, D. Rueckert, J.A. Schnabel, and W.J. Niessen: Automatic Construction of Multiple-object Three-dimensional Statistical Shape Models: Application to Cardiac Modeling, IEEE Trans. Med. Imag., 21, (2002) 1151-66.

14. W. T. Tutte: Convex Representations of Graphs, London Math. Soc., III Ser. 10 (1960) 304-320.

15. L. Freitag and P. Plassmann: Local Optimization-based Simplicial Mesh Untangling and Improvement. Intl. J. of Num. Meth. in Engr., 49 (2000) 109-125.

16. S.A. Canann, J.R. Tristano and M.L. Staten: An Approach to Combined Laplacian and Optimization-Based Smoothing for Triangular, Quadrilateral and QuadDominant Meshes, 7th Int. Meshing Roundtable, (1994) 479-494.

17. X. Gu, Y. Wang, T.F. Chan, P. Thompson and S. Yau: Genus Zero Surface Conformal Mapping and Its Application Surface Mapping, IPMI (2003) 172-184.

18. R.H. Davies, C.J. Twinig, P.J. Allen, T.F Coots and C.J. Taylor: Shape Discrimination in the Hippocampus using an MDL Model, IPMI (2003) 38-50. 